\title{
Community-Arbeit aus der Krise
}

\author{
Oder: Wann ist Krise und wenn ja, für wen?
}

Im Zuge der politischen Aufarbeitung der sogenannten „Flüchtlingskrise” sind auch die Fördermittel für das ehrenamtliche Engagement anderer migrantisierter Personen massiv ausgebaut worden. Eine Entwicklung, die nicht nur positiv bewertet werden kann, sondern ein mangelndes Bewusstsein für Rassismus und Intersektionalität offenbart.

$\mathrm{D}$ ie Gründung des ehrenamtlichen DEMASKKollektivs Ende 2018 kann aus Sicht der Mitglieder durchaus als Reaktion auf einen dauerhaften Krisenzustand verstanden werden. Das queerfeministische intersektionale Kollektiv von und mit Black and People of Color (BPoC) setzt sich durch Community-Arbeit für Repräsentation, Empowerment und gesellschaftliche Teilhabe von Queer/trans*/inter* Black/Indigenous*/People of Color ( $\left.\mathrm{qt}^{*} \mathrm{i} * \mathrm{BI} * \mathrm{PoC}\right)$ ein. Die so verstandene Krise beinhaltet nicht nur das alltägliche Betroffen-sein von Rassismus, Queer- und Trans*-Feindlichkeit, Heteronormativität und Sexis-

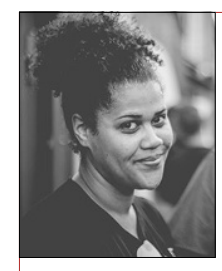

\section{Ella O'Brien-Coker}

Hochschule für Musik und Tanz Köln, Köln, Deutschland *1990; Studium Jazzgesang und Musikerziehung/Vokalpädagogik (B.A.), z. Z. als Studierende des Master Musikwissenschaft und studentische Hilfskraft an der Hochschule für Musik und Tanz Köln . Sängerin, Rapperin, Songwriterin, Mitglied bei DEMASK seit 2019.

ellaobriencoker@gmail.com

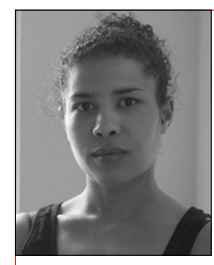

\section{Noelle O'Brien-Coker}

Köln, Deutschland

*1987; Studium Journalistik (M.A.), Philosophie und Musikwissenschaft (B.A.), freie Redakteurin und Autorin, hauptsächlich für den WDR-Hörfunk (COSMO, WDR3, WDR5), Mitglied bei DEMASK seit 2018.

noelle.obriencoker@yahoo.de

Zusammenfassung Ob ein Zustand als Krise wahrgenommen wird, hängt u. a. von der Position derjenigen ab, die sie ausrufen. Für Perspektiven, die von der weißen und cisheteronormativen Mehrheitsgesellschaft abweichen, kann dies bedeuten, dass für sie zentrale Krisenzustände nicht ausreichend oder nachhaltig von Verantwortungsträger_innen adressiert werden.

Schlüsselwörter Community-Arbeit, Rassismus, Heteronormativität, Queer-Feindlichkeit, Intersektionalität, DEMASK mus, sondern auch das Fehlen von Räumen, die die Verschränkungen dieser Marginalisierungsformen ins Zentrum rücken. Derartige Räume möchte DEMASK schaffen, indem die Gruppe kulturelle und politische Veranstaltungen und Workshops für $\mathrm{qt}^{*} \mathrm{i} * \mathrm{BI}$ *PoC organisiert und umsetzt. Mit der ersten Veranstaltungsreihe, dem DEMASK-Fest 2019, fand das Kollektiv großen Anklang in der Community. Spätestens hier zeigte sich, dass der Bedarf nach einem solchen Angebot deutlich größer ist als angenommen.

\section{Intersektionale Netzwerke}

DEMASK spricht von sich und seiner Zielgruppe als Community und von dem, was sie tun, als Community-Arbeit. Der Begriff Community ist von der verbreiteten Bedeutung im englischsprachigen Raum als Nachbarschaft/Nachbarschaftshilfe (Cobigo et al. 2016, S. 188-190) abzugrenzen. Weniger als eine räumliche Nähe wird hier die Identifikation über politische Dimensionen (bspw. das Teilen von Marginalisierungserfahrungen) zentriert. Die Community-Work, der sich DEMASK widmet, unterscheidet sich auch von der professionalisierten Sozialen Arbeit (DBSH 2016) und kann im deutschsprachigen Kontext dem Ehrenamt bzw. bürger_innenschaftlichem Engagement (Weber 2020, S. 4-6) zugeordnet werden: Alle bisherigen Mitglieder betätigen sich unentgeltlich im Kollektiv, gehen einer anderen hauptberuflichen Lohnarbeit nach, die außerhalb der Sozialen Arbeit zu verorten ist oder befinden sich noch in Ausbildung oder Studium.

Was wir als Krise verstehen, mag nicht für alle offenkundig sein. Jedoch ist der Krisenbegriff nicht festgelegt und wird schnell als Überbegriff für verschiedene negative Entwicklungen eingesetzt. Die unklare Definition lässt weiterhin offen, „wann eine Krise beginnt und wann sie endet. Grenzlinien zwischen dem Normalzustand und einer Krise werden nicht weiter präzisiert" 
(Merkel 2015, S. 21). Der Begriff wird weitestgehend zwar als temporär definiert, was sich aber in der vielfältigen Nutzung nicht notwendigerweise widerspiegelt (vgl. ebd.). Die inflationäre Verwendung sorgt außerdem dafür, dass die Schwere einer Krise nicht eindeutig zu identifizieren ist und zudem wird verschleiert, für wen diese Schwere gilt. So ergibt sich für das Kollektiv eine kritische Auseinandersetzung mit dem von uns als herkömmlich wahrgenommenen Verständnis des Begriffs.

Das Erleben von Krise ist zunächst eine Frage der Perspektive: Wir sehen etwa den Kern der sogenannten „Flüchtlingskrise“ nicht darin, dass Schutzsuchende, die vor Krieg und schlechten Lebensbedingungen fliehen, nach Deutschland kommen, sondern vielmehr darin, dass diese Flucht kriminalisiert wird und Menschen im Zuge dessen ihre Rechte abgesprochen werden. Fluchtursachen werden im öffentlichen Diskurs kaum problematisiert, während diese für viele geflüchtete Menschen die eigentlichen Krisen darstellen dürften. Dies zeigt, wie stark es von der Perspektive abhängt, wann eine Krise wahrgenommen und ausgerufen wird.

Die Auswirkungen von Krisen auf Communities und Individuen unterscheiden sich entlang der Dimension gesellschaftlicher Marginalisierung. So begreifen wir als übergeordnete Krise den Dauerzustand von Rassismus, Heteronormativität und Queer- und Trans*-Feindlichkeit. Allein Rassismus formt die Leben direkt Betroffener stark (vgl. Kilomba 2013, S. 104; hooks 2015, S. 170-172; Lorde 2018, S. 44). Die tragende Rolle dieser Erfahrung wird für die Mehrheitsgesellschaft häufig leider erst dann erkennbar, wenn Menschen aufgrund von Rassismus den Tod finden. So erhielt das Thema durch die weltweiten BLM-Proteste große Aufmerksamkeit, allerdings bleibt offen, inwiefern ein echtes nachhaltiges Verständnis der Problematik die Mehrheitsgesellschaft tatsächlich durchdrungen hat ${ }^{1}$. Wir sehen als Betroffene in der Diskussion um die Auswirkungen von Rassismus, dass noch um die Anerkennung des Problems gekämpft werden muss - häufig sogar noch um die Frage, ob es Rassismus in der Mitte der Gesellschaft überhaupt gibt oder nicht. Dies erschwert das Abbauen von rassistischem Denken und Handeln an sich. So verstehen wir Rassismus, sowie andere Ismen, Queer- und Trans*-Feindlichkeit und deren Relativierung als die eigentlich anhaltende Dauerkrise.

\section{Fragwürdige Chancen}

Neben Missverständnissen, Unkenntnis und mangelnder Anerkennung in der Unterhaltung um Rassismus tun sich fragwürdige Chancen auf: Laut einer Anfrage an das Ministerium für Kinder, Familie, Flüchtlinge und
Integration NRW (MKFFI NRW) hat sich seit Beginn der „Flüchtlingskrise“ im Jahr 2014 der Etat für die Förderung der Selbstorganisation von Migrant_innen von 1,2 auf 4,4 Mio. im Förderzeitraum 2021/22 mehr als verdreifacht. Dies greift auch in die Förderstrukturen hinein, in denen sich DEMASK befindet. Demnach wurde die Infrastruktur um Projektförderung für selbstorganisierte Gruppen gestärkt, die sich um die Themen Migration und Integration bemühen. Dem Kollektiv wurde bereits nahegelegt, zwecks eines einfacheren $\mathrm{Zu}$ gangs zu Fördermitteln über die Gründung einer Migrant_innenselbstorganisation (MSO) nachzudenken. Dies ist formell möglich, wird im Kollektiv jedoch kritisch gesehen, da die meisten Mitglieder gar keine Migrant_innen, also selbst nie migriert, sondern in Deutschland geboren und aufgewachsen sind (vgl. Sow 2018, S. 143) und so allenfalls über den sogenannten „Migrationshintergrund" verfügen.

Wir betrachten es als Problem, dass unsere Positionierung nicht differenziert wahrgenommen wird, und dass so unterschiedliche Erfahrungen unter dem Begriff der „Migrant_in“ vereinheitlicht werden, etwa die von Geflüchteten, von ansonsten Migrierten, von Personen mit Migrationshintergrund und denen von BI*PoC. Die Unterschiede zwischen diesen überaus differierenden, sich manchmal durchaus überschneidenden Erfahrungen münden in verschiedenen Bedarfen. Die Positionierung als $\mathrm{BPoC}$ - wie in unserem Fall - soll die gemeinsame Rassismuserfahrung zentrieren und lässt bewusst $\mathrm{Na}$ tionalität oder (primäre) Migrationserfahrung außen vor. Beide Aspekte entsprechen eher der Perspektive der Mehrheitsgesellschaft und einer Haltung, die Integration zunächst in der Verantwortung der ,abweichenden' und $\mathrm{zu}$ integrierenden Individuen legt und die Bekämpfung von Rassismus auf der anderen Seite nicht im selben Maße ernst nimmt (vgl. Gyamerah zit. n. DLF 2021). Integration ist jedoch weder unser Anliegen noch kann sie überhaupt als Forderung an uns als mehrheitlich in Deutschland sozialisierte Personen herangetragen werden. Unsere Privilegien einer deutschen Sozialisierung und eines deutschen Passes lassen uns also daran zweifeln, dass unsere Identität und damit die Intentionen unserer Arbeit - Empowerment und Bekämpfung von Rassismus - tatsächlich wahrgenommen werden. Da es eine wichtige Rolle in unserer Arbeit spielt, Sichtbarkeit und dadurch eine Stimme für die Bedürfnisse unserer Community zu schaffen, kann sich diese fehlende Differenzierung auf verschiedenen Ebenen negativ auswirken. Besonders hinsichtlich einer langfristigen Dekonstruktion von rassistischen Strukturen, ist eine ungenaue Fremdzuschreibung ein Schritt in die entgegengesetzte Richtung. Nicht zuletzt tut sich für uns die 
Frage auf, ob wir mit der Gründung einer MSO einen Raum einnehmen, der uns aufgrund unserer Privilegien nicht zustehen sollte. Weiter führt die Vermischung bzw. Vereinheitlichung der verschiedenen Erfahrungen schlussendlich zu einer Unterwanderung unserer eigenen politischen Agenda zugunsten einer finanziell stabilen Infrastruktur für unsere Community. Ganz zu schweigen von unserer intersektionalen Perspektive: sich überschneidende Erfahrungen als queere, nicht-cismännliche und $\mathrm{BI}$ *PoC werden bei der ausschließlichen Ansprache als MSO vollständig übersehen.

Die Folgen der sogenannten „Flüchtlingskrise“ erweisen sich somit als zweischneidig für das Kollektiv, das zwar offenbar vom Gesetzgeber mitgemeint ist, mit der Inanspruchnahme von Förderung der unzureichenden Fremdzuschreibung aber implizit zustimmt. Zugleich ist unklar, welche Auswirkungen eine Ansprache durch die Politik mit der selbstgewählten Bezeichnung als ( $\mathrm{qt}^{*}{ }^{\mathrm{i}}$ *) BI*PoC hätte. Denkbar ist, dass die Anerkennung von Teilidentitäten inklusive ihrer Privilegien gegenüber noch stärker marginalisierten Personen (mit Flucht- und/ oder primärer Migrationserfahrung) in den Vordergrund gerückt werden, was zum Ausschluss von Fördermitteln für weniger privilegierte Identitäten führen könnte. Damit würde einer der Hauptgründe für die Entstehung und Aufrechterhaltung des Kollektivs gefährdet. Auch stellt sich die Frage, inwiefern es möglich ist, sich nach einer Zustimmung der Fremdzuschreibung von dieser wieder zu distanzieren oder sie rückgängig zu machen. Dies würde unweigerlich zusätzliche Aufklärungsarbeit bedeuten, um die es im spezifischen Rahmen von DEMASK eigentlich nicht geht. Nach einer Zustimmung müsste jedoch Bildungsarbeit in Bezug auf Rassismus zwangsläufig Teil unserer Arbeit werden, insbesondere gegenüber den beanspruchten Fördermittelstrukturen.

Eine weitere Folge könnte sein, dass zwischen den unterschiedlich marginalisierten Gruppen Konkurrenz um mögliche Fördermittel entsteht, die wiederum durch Privilegien, wie in Deutschland sozialisiert zu sein, entschieden werden. Eine interessante Beobachtung hierbei ist, dass das Problem selbst erneut auf die Betroffenen verlagert würde und so Politik und (weiße) Mehrheitsgesellschaft einer tieferen Auseinandersetzung entgehen können. Demnach zeigte ein derartiger Verlauf, dass die Anerkennung der Selbstidentifikation aus rein extrinsischen Gründen geschehen wäre, ohne ein echtes Verständnis über das emanzipatorische Potenzial von Selbstbezeichnungen zu entwickeln und so die durchaus sehr unterschiedlichen und dennoch miteinander verbundenen Identitäten anzuerkennen. Hier könnte erneut beobachtet werden, wie sich die Problematik durch Vermischung von Themen und Begriffen weiter fort- führt: Rassismus wird als Bereich von Migration und Integration und somit als Aufgabe von migrantisch gelesenen Menschen verstanden. Es sollte unseres Erachtens jedoch Ziel einer sich als progressiv und freiheitlich demokratisch verstehenden Gesellschaft sein, Rassismus als gesamtgesellschaftliche Angelegenheit zu sehen und nicht auf die unmittelbar Betroffenen zurückzuwerfen. Es bleibt zu hoffen, dass Fremd- und Selbstwahrnehmung sich aufgrund eines echten Verständnisses dieser komplexen Realitäten synchronisieren, was wiederum mit einer nachhaltigen Anerkennung der von uns als dauerhaft identifizierten Krise des Rassismus (sowie der Queer- und Trans"-Feindlichkeit und des Sexismus) einhergehen würde. Gleichzeitig könnte ein Verständnis für Mehrfachmarginalisierung entstehen und weiter ausgebaut werden. Das Potenzial hinter dem Konzept von Intersektionalität und die Möglichkeiten, die ein tieferes Nachvollziehen der Theorie, Praxis und Methode nach sich zieht, sollte nicht unerkannt bleiben. Erst wenn neben Rassismus auch andere Marginalisierungsformen mit gesehen und gedacht werden, kann sich die zur äußerlichen Vereinfachung gezwungenen Komplexität einer Identität entfalten. So lassen sich nicht nur, wie oben beschrieben, besser Bedarfe benennen, sondern genauso Expertisen und Kompetenzen. Die umfassende und konsequente Förderung von Community-Arbeit aller $(\mathrm{qt} * \mathrm{i} *) \mathrm{BI} * \mathrm{PoC}$ wäre die erhoffte Konsequenz eines solchen gesellschaftspolitischen Erkenntnisprozesses.

\section{Unzureichende Planbarkeit als Zeugnis mangelnden Reformwillens}

Eine für uns zentrale Erkenntnis zum bestehenden Krisenbegriff ist, dass akute Krisen teils übermäßige $\mathrm{Zu}$ wendung erfahren, während latente Krisen häufig unbearbeitet bleiben. Dies wird begleitet von einer eigentlich unannehmbaren Unsicherheit durch limitierte Mittel: Es ist nie ganz sicher, wann eine Zuwendung endet. Fördermittel sind meist abhängig von äußeren politischen Faktoren, wie der Regierungsparteien zum Zeitpunkt der Antragstellung. Wenn Förderperioden enden, ist es letztendlich vom Wohlwollen und der politischen Agenda der neuen Koalition abhängig, ob eine Förderung weitergeht. Gerade jedoch Themen um Menschenrechte (und um nichts Anderes handelt es sich, wenn wir über Rassismus oder Trans*- und Queer-Feindlichkeit sprechen) sind solche Themen, die nicht vom Wohlwollen einzelner Parteien abhängen, sondern institutionell und gesetzlich verankert sein sollten.

Die Hoffnung bleibt. Wenn es gelingen kann, die Missverständnisse zu erkennen und die Stimmen von ( $\mathrm{qt} * \mathrm{i} *$ ) $\mathrm{BI}$ *PoC aktiv in den Diskurs mit einzubeziehen, gibt es eine klare Chance die formulierten Probleme zu bewäl- 
tigen. Ausdrücklich wäre es zu begrüßen, ein übergeordnetes Eingeständnis gegenüber verschiedenen rassistischen, bzw. intersektional marginalisierten Erfahrungen erkannt zu sehen, das unabhängig von Wahlergebnissen bestehen bleibt. Denn so kann auf einer nachhaltigen Basis an tief verankerten Problemen gearbeitet werden. Eine Kontinuität im Erkennen und Anerkennen der rassistischen Realität für BI*PoC zeugt nicht nur von Integrität, sondern fördert Respekt, den viele von Diskriminierung Betroffene vermissen. Ein Verständnis davon, dass ein ernstzunehmendes Angehen der bestehenden Verhältnisse ein Prozess bleibt, kann den Handlungsspielraum Einzelner vergrößern und die Ohnmacht gegenüber systematischen Zusammenhängen verringern. Abschließend können wir festhalten, dass eine Anerkennung von ( $\left.\mathrm{qt}^{*} \mathrm{i} *\right)$ BI*PoC und anderer zusammenlaufender Erfahrungen jenseits der weißen Mehrheitsgesellschaft mit der Anerkennung übergeordneter Krisen (Rassismus, Queer- und Trans*-Feindlichkeit usw.) einhergeht. Eine Verschiebung des Diskurses von der Frage, „ob es Rassismus wirklich gibt“, hin zu einer Denkweise, die die Auswirkungen von Rassismus erkennt und benennt, hat das Potenzial, hierarchische und so eben auch rassistische Strukturen aufzuweichen. Ein fairer und informierter Umgang mit marginalisierten Identitäten, sowie das Identifizieren und Transparent-Machen von Privilegien sind gute Gründe, sich die Mühe zu machen. Nicht zuletzt, um selbst an diesen Aufgaben wachsen zu können.

Eingegangen. 31. März 2021

Angenommen. 26. April 2021

Funding. Open Access funding enabled and organized by Projekt DEAL.

Open Access. Dieser Artikel wird unter der Creative Commons Namensnennung 4.0 International Lizenz veröffentlicht, welche die Nutzung, Vervielfältigung, Bearbeitung, Verbreitung und Wiedergabe in jeglichem Medium und Format erlaubt, sofern Sie den/die ursprünglichen Autor(en) und die Quelle ordnungsgemäß nennen, einen Link zur Creative Commons Lizenz beifügen und angeben, ob Änderungen vorgenommen wurden.

Die in diesem Artikel enthaltenen Bilder und sonstiges Drittmaterial unterliegen ebenfalls der genannten Creative Commons Lizenz, sofern sich aus der Abbildungslegende nichts anderes ergibt. Sofern das betreffende Material nicht unter der genannten Creative Commons Lizenz steht und die betreffende Handlung nicht nach gesetzlichen Vorschriften erlaubt ist, ist für die oben aufgeführten Weiterverwendungen des Materials die Einwilligung des jeweiligen Rechteinhabers einzuholen.

Weitere Details zur Lizenz entnehmen Sie bitte der Lizenzinformation auf http://creativecommons.org/licenses/by/4.0/deed.de.
1. Wie die Debatte um rassistische Sendungen im WDR-Fernsehen zeigte. Dass die Kritik daran überhaupt ein so großes Echo erhalten konnte, sehen wir wiederum als Fortschritt, den wir uns mit der breiten Auseinandersetzung mit Rassismus in 2020 erklären.

\section{Literatur}

Cobigo, V., \& Mcheimech Rawad, M. L. (2016). Understanding community. Canadian journal of disability studies. https://cjds.uwaterloo.ca/index. php/cjds/article/view/318/540. Zugegriffen: 28. März 2021.

Deutscher Berufsverband für Soziale Arbeit e. V. - DBSH (2016). Deutschsprachige Definition Sozialer Arbeit. Berlin. https://www.dbsh.de/media/ dbsh-www/redaktionell/bilder/Profession/20161114_Dt_Def_Sozialer_Arbeit_FBTS_DBSH_01.pdf. Zugegriffen: 28. März 2021.

DLF (2021). Vor Integrationsgipfel: Interview Daniel Gyamerah, Vorstand Each-One-Teach-One. In Informationen am Morgen, Sendedatum: 09.03.2021. https://ondemand-mp3.dradio.de/file/dradio/2021/03/09/ vor_integrationsgipfel_interview_daniel_gyamerah_vorstand_ dlf_20210309_0820_b4e68b6b.mp3. Zugegriffen: 30. März 2021.

hooks, (2015). Black Looks. Race and Representation. New York: Routledge.

Kilomba, G. (2013). Plantation memories. Episodes of everyday racism. Münster: UNRAST.

Lorde, A. (2018). The master's tools will never dismantle the master's house. Milton Keynes: Penguin Random House.

Merkel, W. (2015). Die Herausforderungen der Demokratie. In W. Merkel (Hrsg.), Demokratie und Krise (S. 7-42). Wiesbaden: Springer.

Sow, N. (2018). Deutschland Schwarz Weiß. Der alltägliche Rassismus. Norderstedt: BoD Books on Demand.

Weber, U. (2020). Bürgerschaftliches Engagement. Eine Einführung. In K. Grunwald \& L. Kollhoff (Hrsg.), Basiswissen Sozialwirtschaft und Sozialmanagement (S. 4-6). Wiesbaden: Springer.

\section{Weiterführende Literatur}

van Dyk, S. (2019). Identitätspolitik gegen ihre Kritik gelesen. Für einen rebellischen Universalismus. In Bundeszentrale für politische Bildung (Hrsg.), Aus Politik und Zeitgeschehen (S. 25-32). Bonn: Bundeszentrale für politische Bildung. 